\title{
FREQUÊNCIA FUNDAMENTAL DAS 7 VOGAIS ORAIS DO PORTUGUÊS EM VOZES DE CRIANÇAS
}

\author{
Fundamental frequency of seven oral vowels of Portuguese \\ in children voices
}

Flávia Viegas ${ }^{(1)}$, Danieli Viegas ${ }^{(2)}$, Ciríaco Cristovão Tavares Atherino ${ }^{(3)}$, Heidi Elisabeth Baeck ${ }^{(4)}$

\begin{abstract}
RESUMO
Objetivo: investigar as medidas de frequência fundamental das 7 vogais orais do português em crianças saudáveis entre 4 e 8 anos. Métodos: foram selecionadas através de triagem da expressão oral e avaliação perceptivo-auditiva da voz, 207 crianças de ambos os sexos, que foram divididas por faixa etária. Os sinais de fala foram obtidos a partir das gravações de sentenças-veículo. Foram selecionados segmentos das 7 vogais orais do português falado no Brasil em posição tônica para a estimação das medidas de frequência fundamental. O recorte e processamento dos sinais foram realizados com o auxílio do software Praat. Resultados: os achados foram expostos por meio de valores médios do parâmetro investigado, sendo observado um decréscimo na frequência fundamental com o aumento da idade. A idade de seis anos foi apontada como determinante para as mudanças acústicas das vocalizações infantis. Conclusão: os valores paramétricos apontados consistem em uma importante contribuição para a língua portuguesa falada no Brasil. A idade de seis anos deve ser considerada em futuros trabalhos que investiguem vozes infantis
\end{abstract}

DESCRITORES: Voz; Processamento de Sinais Assistido por Computador; Criança; Acústica da Fala

\section{INTRODUÇÃO}

O século XX marcou o período moderno da avaliação vocal e os avanços tecnológicos alcançados propiciaram a intensificação das pesquisas de análise da voz ${ }^{1,2}$. Sistemas baseados em circuitos analógicos de tempo contínuo passaram a

(1) Fonoaudióloga da Prefeitura da Cidade do Rio de Janeiro, PCRJ, Rio de Janeiro, RJ; Mestre em Fonoaudiologia pela Universidade Veiga de Almeida.

(2) Fonoaudióloga da Prefeitura da Cidade do Rio de Janeiro, PCRJ, Rio de Janeiro, RJ; Mestre em Fonoaudiologia pela Universidade Veiga de Almeida.

(3) Médico; Professor Adjunto do Mestrado Profissionalizante em Fonoaudiologia da Universidade Veiga de Almeida, UVA, Rio de Janeiro, RJ; Professor Adjunto da disciplina de Otorrinolaringologia da Faculdade de Ciências Médicas da Universidade Estadual do Rio de Janeiro, UERJ, Rio de Janeiro, RJ; Doutor em Otorrinolaringologia pela Faculdade de Medicina da Universidade de São Paulo.

(4) Fonoaudióloga; Professora Adjunta do Mestrado Profissionalizante em Fonoaudiologia da Universidade Veiga de Almeida, UVA, Rio de Janeiro, RJ; Doutora em Engenharia Biomédica pela Universidade Federal do Rio de Janeiro.

Conflito de interesses: inexistente ser implementados através de sistemas digitais de tempo discreto ${ }^{3}$, abrindo a possibilidade de realizar análises vocais quantitativas, essenciais no desenvolvimento de sistemas de reconhecimento automático de padrões vocais ${ }^{4}$, que por sua vez, consistem em bases de ferramentas de auxílio diagnóstico e terapêutico.

Apesar da diversidade de possibilidades de aplicação da análise vocal digital, pode ser observada na literatura uma tendência de se investigar parâmetros acústicos em uma população predominantemente adulta ${ }^{5-12}$. A população infantil é pesquisada de forma mais restrita. A relevância de se buscar a padronização de parâmetros acústicos da voz infantil pode ser apontada, no mínimo, em duas direções: a primeira está associada ao potencial de aplicação de tais padrões no campo do auxílio diagnóstico de transtornos/distúrbios vocais na infância; enquanto a segunda associa-se à necessidade de tais padrões no suporte às pesquisas que investigam a correlação entre as oralizações de bebês e o desenvolvimento dos movimentos articulatórios na criança. Em comum, os autores desta segunda linha de pesquisa, enfatizam a carência na 
literatura de medidas acústicas referenciais da voz infantil ${ }^{13,14}$.

Nas pesquisas em que a frequência fundamental da voz normal infantil foi contemplada, pode ser observada uma tendência de investigação de apenas determinadas vogais, como a vogal [a] ${ }^{15-19}$ e a vogal $[\varepsilon]^{20,21}$. As demais vogais não recebem a mesma atenção por parte dos pesquisadores. Dentre os estudos realizados na língua portuguesa, é possível observar algumas pesquisas em que o número de sujeitos investigados foi em torno de $30{ }^{15,17}$ e outras, com número em torno de $100^{20,21}$. No presente trabalho, optou-se pela contemplação de uma amostra mais significativa em termos de número de sujeitos e de vogais. O objetivo deste estudo foi investigar as medidas de frequência fundamental das 7 vogais orais do português em crianças saudáveis entre 4 e 8 anos visando contribuir para a determinação de padrões acústicos vocais infantis do português brasileiro.

\section{MÉTODOS}

O presente estudo, de tipo coorte transversal, apresentou amostra constituída por 1.449 segmentos vocais de 207 crianças de ambos os sexos entre quatro e oito anos. As crianças foram distribuídas por grupos de acordo com as seguintes faixas etárias: G1 (4-5 anos), G2 (5-6 anos), G3 (6-7 anos) e G4 (7-8 anos).

Duzentos e trinta e duas crianças foram pré-selecionadas nos setores de pediatria e odontologia da Policlínica Maria Cristina Roma Paugartten (Secretaria Municipal de Saúde da Prefeitura do Rio de Janeiro) e em duas escolas, pelos profissionais de saúde e educação destas unidades. Estes profissionais foram instruídos a encaminhar para o setor de fonoaudiologia da referida policlínica crianças que apresentassem condições de expressão oral e saúde geral dentro dos padrões de normalidade. No setor de fonoaudiologia, as crianças encaminhadas foram submetidas aos procedimentos de seleção propriamente ditos e os pais ou responsáveis assinaram o Termo de Consentimento Livre e Esclarecido. Elas passaram por uma triagem da expressão oral através do Exame Fonético Realfa, cujo objetivo foi a exclusão das que apresentavam expressão oral incompatível com os padrões esperados para a faixa etária.

As crianças incluídas nesta fase tiveram a fala espontânea e repetição de sentenças-veículo gravadas. A fala espontânea deu suporte à análise perceptivo-auditiva da voz, que contemplou parâmetros relativos à fonte glótica e à ressonância. Nesta análise, utilizaram-se a Escala RASATI ${ }^{22}$ (Figura 1) e um roteiro de avaliação do sistema ressonantal ${ }^{23}$
(Figura 2). Foram incluídas as crianças que apresentaram padrões adequados em ambas as avaliações. Consideraram-se adequadas na avaliação da fonte glótica (Escala RASATI) vozes que apresentaram grau 0 nos seis itens avaliados, com exceção do item soprosidade, que poderia apresentar grau 0 (ausente) ou 1 (leve). Esta tolerância foi permitida uma vez que a população infantil pode apresentar soprosidade em grau 1 como padrão esperado ${ }^{20,24}$. $\mathrm{Na}$ avaliação de ressonância, foram consideradas as vozes que apresentaram ressonância equilibrada. Os informantes incluídos na fase perceptivo-auditiva, passaram para a fase de análise de sentenças-veículo. Nas duas fases de seleção, 25 crianças foram excluídas por inadequação da expressão oral ou da voz.

A sentença-veículo ("Fale___ para mim") foi preenchida com os vocábulos "pápa", "pépe", "pêpe", "pípi", "pópo", "pôpo" e "púpu". Estas emissões foram gravadas em uma sala silenciosa, em mono canal, com uma taxa de amostragem de $22.050 \mathrm{~Hz}$, em formato ".wav". Os materiais utilizados foram o software Praat (versão 5008), um notebook marca HP (modelo DV 1000), com sistema operacional Windows XP e um microfone da marca SHURE (modelo SM 58), posicionado a distância de $10 \mathrm{~cm}$ dos lábios da criança.

Foram selecionados segmentos dos vocábulos das sete vogais orais do português em posição tônica para a estimação das medidas de frequência fundamental $\left(f_{0}\right)$. Os dados foram submetidos a procedimentos de pré-processamento (recorte dos segmentos vocálicos), processamento (estimação de parâmetros acústicos) e análise estatística. O pré-processamento e processamento foram realizados com auxílio do software Praat, enquanto que a análise estatística foi realizada com o software SAS 6.04 (SAS Institute, Inc., Cary, North Carolina).

O recorte das vogais foi realizado de forma manual, porém houve a preocupação em definir e seguir critérios de recorte passíveis de serem implementados em um futuro sistema de recorte automático. O marco inicial foi definido no trecho do sinal onde a energia apresentava-se acima de $20 \%$ da energia máxima. Da mesma forma, o marco final foi estabelecido onde a energia apresentava-se abaixo de $20 \%$ da mesma. Tal porcentagem foi estabelecida empiricamente com base em investigação experimental. Este procedimento é conservador no sentido de reduzir a probabilidade de obtenção de trechos não estacionários.

Devido ao grande número de estimações envolvidas, foi utilizado um script (ferramenta que extrai automaticamente, de forma padronizada, as medidas paramétricas) criado pelos autores, que viabilizou a otimização do tempo de processamento 


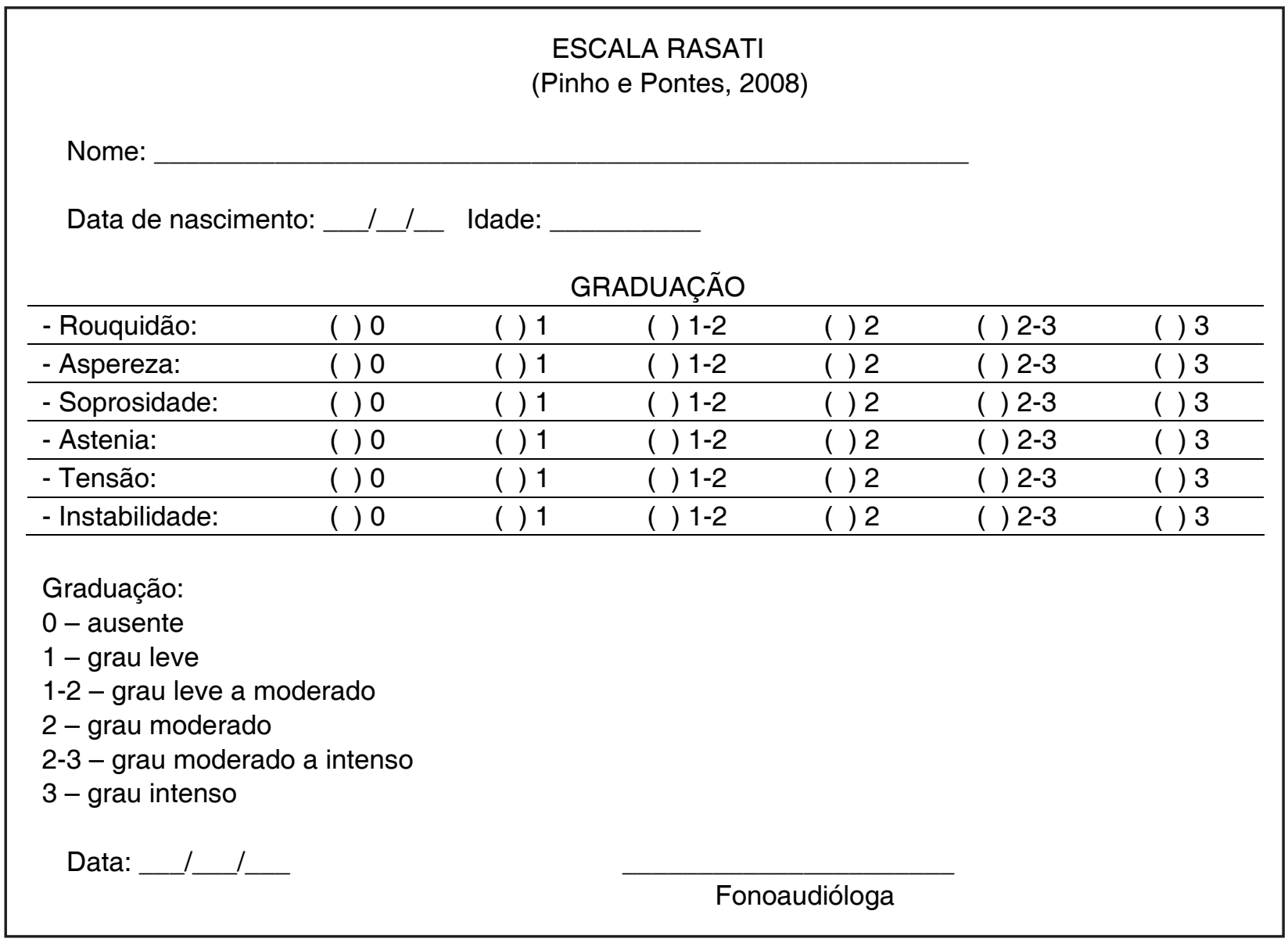

Figura 1 - Escala RASATI (Pinho e Pontes, 2008) ${ }^{22}$

\section{ROTEIRO DE AVALIAÇÃO DO SISTEMA RESSONANTAL}

(Pinho, 2003)

FOCO DE RESSONÂNCIA:

a) ( ) nasal

Tipo: ( ) rinofonia aberta ( ) rinofonia fechada

( ) hiponasalidade ( ) denasalidade

b) ( ) faríngeo

c) ( ) cul de sac

d) ( ) laringo-faríngeo

e) ( ) equilibrado

Data:

Fonoaudióloga

Figura 2 - Roteiro de avaliação do sistema ressonantal (Pinho, 2003) ${ }^{23}$ 
e evitou possíveis erros de manuseio na sequência dos procedimentos de estimação.

A presente pesquisa foi aprovada pelo Comitê de Ética em Pesquisa da Secretaria Municipal de Saúde da Prefeitura do Rio de Janeiro (n 180/2008).

$\mathrm{Na}$ análise estatística, foram utilizados os seguintes testes estatísticos: o teste de MannWhitney para comparação das medidas de frequência fundamental entre os sexos e análise de variância de Kruskal-Wallis e o teste de Comparações Múltiplas de Duncan para comparação entre as quatro faixas etárias pesquisadas. A opção pela utilização de teste não paramétrico foi pelo fato de que a maioria das medidas de frequência não apresentou distribuição normal (Gaussiana), devido à dispersão dos dados, falta de simetria da distribuição e pela rejeição da hipótese de normalidade, segundo o teste de Shapiro-Wilk (W). O critério de determinação de significância adotado foi o nível de $5 \%$, ou seja, valor de $p$ menor ou igual a 0,05 .

\section{RESULTADOS}

Os achados da presente pesquisa encontramse resumidos nas Tabelas 1 a 3 . São expostos os valores médios referentes à frequência fundamental e seus respectivos desvios-padrão em função do grande número de estimações obtidas neste trabalho, um total de 2.898 valores numéricos.

A distribuição dos informantes por faixa etária e sexo é apresentada na Tabela 1. Na Tabela 2, são apresentadas as médias e desvios-padrão de frequência fundamental das 7 vogais orais do Português falado no Brasil, de acordo com o sexo e faixa etária.

Na Tabela 3 encontram-se expostos os achados referentes aos testes de diferença estatística em relação aos sexos e faixas etárias.

No teste de Mann-Whitney foram observadas diferenças entre os sexos com nível de significância de $5 \%$ nas vogais [e], [ó] e [o].

$\mathrm{Na}$ análise de variância de Kruskal-Wallis foram observadas diferenças estatísticas com nível de significância de $5 \%$ em todas as vogais analisadas. No teste de Comparações Múltiplas de Duncan foi observada uma clara diferença entre os grupos etários G1 (4-5 anos) e G4 (7-8 anos). Ao comparar os grupos etários G1 (4-5 anos) com G3 (6-7 anos) e G2 (5-6 anos) com G4 (7-8 anos), observou-se diferenças em algumas vogais. No entanto, não

Tabela 1 - Distribuição dos informantes por faixa etária e sexo

\begin{tabular}{ccccc}
\hline & $4-5$ anos & 5-6 anos & 6-7 anos & 7-8 anos \\
\hline Masculino & $\mathrm{n}=22$ & $\mathrm{n}=28$ & $\mathrm{n}=24$ & $\mathrm{n}=24$ \\
Feminino & $\mathrm{n}=21$ & $\mathrm{n}=33$ & $\mathrm{n}=29$ & $\mathrm{n}=26$ \\
\hline
\end{tabular}

Tabela 2 - Médias e desvios-padrão de frequência fundamental das 7 vogais orais do Português de acordo com o sexo e faixa etária

\begin{tabular}{|c|c|c|c|c|c|c|c|c|c|}
\hline \multirow{2}{*}{ Parâmetros } & \multirow{2}{*}{ Sexo } & \multicolumn{2}{|c|}{ 4-5 anos } & \multicolumn{2}{|c|}{ 5-6 anos } & \multicolumn{2}{|c|}{ 6-7 anos } & \multicolumn{2}{|c|}{$7-8$ anos } \\
\hline & & ME & (土DP) & ME & ( $\pm D P)$ & ME & ( $\pm \mathrm{DP})$ & ME & $( \pm D P)$ \\
\hline \multirow{2}{*}{ Vogal [a] } & masc & 270 & 26,1 & 259 & 24,5 & 258 & 25,3 & 243 & 22,4 \\
\hline & fem & 259 & 24,1 & 247 & 22,1 & 256 & 27,5 & 246 & 28,0 \\
\hline \multirow{2}{*}{ Vogal $[\varepsilon]$} & masc & 266 & 21,5 & 248 & 34,2 & 266 & 21,9 & 245 & 23,5 \\
\hline & fem & 258 & 19,1 & 250 & 19,1 & 252 & 23,9 & 246 & 29,0 \\
\hline \multirow{2}{*}{ Vogal $[e]$} & masc & 275 & 24,5 & 258 & 25,3 & 271 & 20,9 & 252 & 25,1 \\
\hline & fem & 267 & 15,1 & 252 & 18,3 & 260 & 24,1 & 247 & 27,3 \\
\hline \multirow{2}{*}{ Vogal [i] } & masc & 301 & 30,8 & 279 & 30,6 & 288 & 24,1 & 275 & 34,0 \\
\hline & fem & 287 & 30,2 & 276 & 22,7 & 282 & 27,3 & 272 & 37,3 \\
\hline \multirow{2}{*}{ Vogal [ó] } & masc & 271 & 24,2 & 254 & 26,4 & 263 & 19,9 & 256 & 26,1 \\
\hline & fem & 256 & 24,2 & 250 & 18,0 & 255 & 25,6 & 251 & 26,7 \\
\hline \multirow{2}{*}{ Vogal [o] } & masc & 281 & 26,3 & 261 & 22,7 & 267 & 22,4 & 257 & 23,8 \\
\hline & fem & 259 & 40,5 & 260 & 21,0 & 260 & 23,2 & 253 & 30,4 \\
\hline \multirow{2}{*}{ Vogal [u] } & masc & 313 & 35,5 & 282 & 40,4 & 299 & 34,8 & 279 & 26,5 \\
\hline & fem & 295 & 25,9 & 298 & 39,9 & 284 & 27,8 & 277 & 40,7 \\
\hline
\end{tabular}


Tabela 3 - Comparações entre os sexos e entre as faixas etárias das medidas de frequencia fundamental

\begin{tabular}{|c|c|c|c|c|c|c|c|c|}
\hline \multirow{2}{*}{$\begin{array}{c}\mathbf{f}_{0} \\
\text { Vogal }\end{array}$} & \multirow{2}{*}{$\begin{array}{c}\text { Sexo } \\
p \text { valor }\end{array}$} & \multirow{2}{*}{$\begin{array}{l}\text { Faixas } \\
\text { etárias } \\
p \text { valor }^{b}\end{array}$} & \multicolumn{6}{|c|}{ Comparações Múltiplas de Duncan } \\
\hline & & & G1 $\times$ G2 & G1 x G3 & G1 x G4 & G2 x G3 & G2 x G4 & G3 x G4 \\
\hline [a] & 0,099 & $0,001^{*}$ & & * & * & & * & \\
\hline$[\varepsilon]$ & 0,092 & $0,0003^{*}$ & & * & * & & * & \\
\hline$[\mathrm{e}]$ & $0,038^{*}$ & $0,0001^{*}$ & & * & * & * & * & \\
\hline [i] & 0,081 & $0,003^{*}$ & & * & * & & & \\
\hline [ó] & $0,013^{*}$ & $0,050^{*}$ & & * & * & & & \\
\hline [o] & $0,046^{*}$ & $0,008^{*}$ & & & * & & & \\
\hline$[\mathrm{u}]$ & 0,42 & $0,008^{*}$ & & & * & & & \\
\hline
\end{tabular}

a nível descritivo do teste de Mann-Whitney; ${ }^{b}$ nível descritivo da ANOVA de Kruskal-Wallis; ${ }^{*}$ expressa diferença significativa, ao nível de 5\%; G1=4 a 5 anos; G2=5 a 6 anos ; G3=6 a 7 anos; G4=7 a 8 anos.

foi observada nenhuma diferença estatisticamente significante entre os grupos G1 (4-5 anos) e G2 (5-6 anos) e, também entre os grupos G3 (6-7 anos) e G4 (7-8 anos). Desta forma, esses dados apontam para o agrupamento de $\mathrm{G} 1 \mathrm{com} \mathrm{G} 2$, e de $\mathrm{G} 3 \mathrm{com}$ G4, sendo o marco etário entre esses grupos a idade de 6 anos.

\section{DISCUSSÃO}

A presente discussão foi desenhada de forma a contemplar duas vertentes principais: as medidas de frequência fundamental da voz infantil e a hipótese da existência de diferenças paramétricas atribuídas à faixa etária e ao sexo da criança.

\section{Medidas de frequência fundamental da voz infantil}

A literatura que investiga a voz infantil saudável apresenta publicações que contemplam a vogal $[a]^{15-19} \mathrm{e}$, em menor número, a vogal $[\varepsilon]^{20,21}$. De forma geral, os valores obtidos para a vogal $[a]$ e $[\varepsilon]$ no presente trabalho corroboram a literatura.

Dentro da língua portuguesa falada no Brasil, autores ${ }^{17}$ que investigaram a frequência fundamental exclusivamente da vogal [a], emitida por crianças de quatro a seis anos e oito meses, mostraram resultados que foram corroborados pelos presentes achados. Este fato foi observado tanto no valor médio de $\mathrm{f}_{0}$, quanto nos valores do mesmo parâmetro, específicos por faixa etária (quatro, cinco e seis anos) e sexo (masculino e feminino). Ao analisar emissões infantis da vogal [a], uma autora ${ }^{15}$ obteve uma $f_{0}$ média mais grave, entretanto, a faixa etária das crianças de sua amostra (8 a 12 anos) não corresponde à investigada no presente trabalho, inviabilizando, desta forma, a comparação entre os resultados.

Autores internacionais também mantiveram o foco sobre a frequência fundamental da vogal [a]. Ao contemplar vozes de crianças entre seis e doze anos, pesquisadores ${ }^{18}$ observaram que a $f_{0}$ decresceu, tanto para os meninos quanto para as meninas. Observou-se que os valores iniciais, que correspondem à faixa etária mais próxima de 6 anos, encontram-se próximos aos resultados apontados no presente trabalho e os valores finais, correspondentes à faixa de doze anos, corroboram os valores obtidos por outra autora ${ }^{15}$. Alguns estudiosos ${ }^{19}$ investigaram a emissão da vogal [a] de forma sustentada em crianças menores. A média de $f_{0}$ encontrada se aproxima do valor mais baixo de $f_{0}$ da vogal [a] obtida no presente trabalho. Outros pesquisadores ${ }^{16}$ apontaram valores médios de $f_{0}$ para os sexos masculino e feminino com base em duas faixas etárias, quatro a seis anos e seis a oito anos. Embora, em princípio, as médias no presente estudo tenham sido expostas em base anual, se os valores forem agrupados, acomodando de forma que se obtenham as mesmas faixas etárias dos autores, poderão ser observados achados basicamente convergentes. No entanto, o valor correspondente ao sexo feminino, na faixa de 6 a 8 anos, apresentou-se sutilmente mais elevado nos achados dos autores.

Assim como a vogal [a], mensurações de $f_{0} d a$ vogal $[\varepsilon]$ na população infantil brasileira também foram apresentadas na literatura. Investigando as mesmas faixas etárias (seis a oito anos), dois grupos de estudiosos ${ }^{20,21}$ apontaram valores médios de $\mathrm{f}_{0}$ diferentes $(249 \mathrm{~Hz}$ e $238 \mathrm{~Hz})$. A média para a vogal $[\varepsilon]$ do presente trabalho $(253 \mathrm{~Hz})$ converge 
com o resultado do primeiro grupo ${ }^{20}$. A comparação entre os dois trabalhos mostrou valores médios de $\mathrm{f}_{0}$ semelhantes, embora a faixa dinâmica obtida pelos autores tenha se mostrado consideravelmente mais larga, tanto no que tange à frequência fundamental mínima quanto à máxima. Na comparação entre os desvios padrão foi observado que os valores divergem consideravelmente, sendo maiores no presente estudo. Enquanto os achados deste trabalho mostram um desvio de $10 \%$ do valor médio, o grupo de autores da literatura mostra um desvio que representa pouco mais de $1 \%$ do valor médio de $\mathrm{f}_{0}$. Não foi possível a comparação dos desvios padrão com o outro trabalho ${ }^{21}$, uma vez que apesar de terem relatado a estimação deste parâmetro, os autores não apresentam os valores obtidos. Embora um desvio padrão reduzido seja desejável em qualquer pesquisa, ao analisar outros estudos de voz pode-se notar que estes apresentam valores de desvio padrão que variam em torno dos $10 \%{ }^{14,15}$.

\section{Diferenças entre faixas etárias e sexos}

\section{Faixas Etárias}

Se observado o conjunto de resultados dos testes estatísticos para as faixas etárias (Tabela 3), pode ser notada a confirmação de diferenças estatísticas em cinco das sete vogais analisadas entre os grupos G1 (4-5 anos) e G3 (6-7 anos), entre três das sete vogais analisadas entre os grupos $\mathrm{G} 2$ (5-6 anos) e G4 (7-8 anos) e apenas uma diferença entre os grupos G2 (5-6 anos) e G3 (6-7 anos). Os testes apontaram ainda diferenças em todas as vogais entre os grupos etários G1 (4-5 anos) e G4 (7-8 anos), mostrando desta forma, a existência de uma diferença clara entre os valores da frequência fundamental das vozes das crianças de 4 a 5 anos e 7 a 8 anos de idade. Não foram observadas diferenças estatísticas entre os grupos G1 (4-5 anos) e G2 (5-6 anos) e entre G3 (6-7 anos) e G4 (7-8 anos), apontando, desta forma, para o agrupamento dos mesmos. Pode ser notado que a idade de seis anos encontra-se na divisão entre os dois grupos, portanto, essa idade parece ser determinante para as mudanças acústicas das vocalizações infantis.

Além do apontamento com relação à idade de 6 anos como marco importante para as mudanças acústicas na voz infantil, os resultados do presente trabalho assinalam, de forma geral, a existência de uma relação decrescente da frequência fundamental com a faixa etária, ou seja, quanto maior a faixa etária, menor a medida de frequência observada. Tal comportamento da frequência fundamental também é descrito na literatura ${ }^{20,25}$.

\section{Sexos}

O teste de diferença estatística entre os sexos apontou diferenças em três das sete vogais analisadas. No entanto, a ocorrência de significâncias estatísticas não se mostrou consistente (Tabela 3) a ponto de que pudesse levar à generalização do conceito de que a frequência fundamental de meninos é diferente de meninas.

$\mathrm{Na}$ literatura, é possível observar que a hipótese da existência de diferenças entre os sexos em parâmetros acústicos vem sendo descrita há anos. Autores ${ }^{26}$ encontraram significância estatística entre os sexos na $f_{0}$ de vozes de crianças de cinco a onze anos, com valores maiores para as meninas. Recentemente, os resultados de uma pesquisa ${ }^{20}$ também apontaram diferenças significantes na $f_{0}$ em crianças de seis a oito anos. No entanto, outros pesquisadores ${ }^{25}$ não encontraram diferenças significativas neste parâmetro ao contemplar crianças de cinco a onze anos.

Alguns autores referiram ter encontrado diferenças entre os sexos apenas após uma determinada idade. Ao avaliar a $f_{0}$ e frequências dos três primeiros formantes, um grupo de autores ${ }^{27}$ apontou diferenças somente a partir dos 11 anos de idade. Outros pesquisadores ${ }^{28}$ concluíram que embora a análise das frequências dos formantes tivesse permitido a diferenciação entre os sexos em crianças a partir de quatro anos de idade, a análise da $f_{0}$ só pôde diferenciar os sexos a partir dos 12 anos.

\section{CONCLUSÃO}

Os resultados deste trabalho apresentam uma contribuição para determinação de padrões vocais infantis do português brasileiro. Assim como assinalado por alguns autores, os presentes achados confirmaram a existência de uma relação decrescente das medidas de frequência fundamental com a idade, ou seja, quanto maior a faixa etária, menores as medidas de frequência fundamental observadas.

Os resultados deste estudo não mostraram diferenças estatísticas entre os grupos etários $\mathrm{G} 1$ (4-5 anos) e G2 (5-6 anos) e, entre G3 (6-7 anos) e G4 (7-8 anos), apontando, para o agrupamento de G1 com G2 e G3 com G4. A idade de seis encontrase no limite entre os dois grupos, desta forma, pode-se concluir que tal idade parece ser determinante para as mudanças acústicas das vocalizações infantis e, deve, portanto, ser considerada nos futuros trabalhos que investiguem vozes infantis. 


\section{ABSTRACT}

Purpose: to investigate fundamental frequency of the 7 oral vowels of Brazilian Portuguese on voices of healthy children between four and eight year old. Methods: two hundred and seven children of both genders were select by speech tasks and by a voice auditory-perceptive analysis. They were separated by age groups. Recordings were obtained from carrier phrases and segments of the seven oral vowels of Brazilian Portuguese in the stressed syllable were selected to estimate the fundamental frequency. Praat Software was used to cut and process these recordings. Results: our findings showed medium values for the parameter investigated and we observed that the fundamental frequency values decreased as age increased. We pointed out that acoustical changes on voice of children can be determinate at the age of six years. Conclusion: The found parametric values consist of an important contribution to the Portuguese language, spoken in Brazil. The age of six years should be considered in futures works that investigate children voices.

KEYWORDS: Voice; Signal Processing, Computer-Assisted; Child; Speech Acoustics

\section{REFERÊNCIAS}

1. Sader RM, Hanayama EM. Considerações teóricas sobre a abordagem acústica da voz infantil. Rev. CEFAC. 2004; 6(3):312-8.

2. Schwarz K, Cielo CA. Modificações laríngeas e vocais produzidas pela técnica de vibração de língua sonorizada. Pró-Fono. 2009; 21(2):161-6.

3. Mitra SK. Digital signal processing: a computerbased approach. New York: McGrawHill; 2001.

4. Baeck HE, Souza MN. A Bayesian classifier for babies' cry pain and non-pain contexts. In: Proceedings of $25^{\text {th }}$ International Conference on the IEEE Engineering in Medicine and Biology Society, Cancun, v.1, p. 2944-6, 2003.

5. Corazza VR, Silva VFC, Queija DS, Dedivitis RA, Barros APB. Correlação entre os achados estroboscópicos, perceptivo-auditivos e acústicos em adultos sem queixa vocal. Rev Bras Otorrinolaringol. 2004; 70(1):30-4.

6. Mccrea CR, Morris, RJ. The effects of fundamental frequency level on voice onset time in normal adult male speakers. J Speech Lang Hear Res. 2005; 48:1013-24.

7. Nemr K, Amar A, Abrahão M, Leite GCA, Köhle $J$, Santos $A O$, et al. Análise comparativa entre avaliação fonoaudiológica perceptivo-auditiva, análise acústica e laringoscopias indiretas para avaliação vocal em população com queixa vocal. Rev Bras Otorrinolaringol. 2005; 71(1):13-7.

8. Gelfer MP, Mikos VA. The relative contributions of speaking fundamental frequency and formant frequencies to gender identification based on isolated vowels. J Voice. 2004; 19(4):544-54.

9. Mifune E, Justino VSS, Camargo Z, Gregio F. Análise acústica da voz do idoso: caracterização da freqüência fundamental. Rev. CEFAC. 2007; 9(2):238-47.

10. Gama ACC, Behlau MS. Estudo da constância de medidas acústicas de vogais prolongadas e consecutivas em mulheres sem queixa de voz e em mulheres com disfonia. Rev Soc Bras Fonoaudiol. 2009; 14(1):8-14.

11. Finger LS, Cielo CA, Schwarz K. Acoustic vocal measures in women without voice complaints and with normal larynxes. Braz $\mathrm{J}$ Otorhinolaryngol. 2009; 75(3):432-40.

12. Cerceau JSB, Alves CFT, Gama ACC. Análise acústica da voz de mulheres idosas. Rev. CEFAC. 2009; 11(1):142-149.

13. Rothgänger $\mathrm{H}$. Analysis of the sounds of the child in the first year of age and a comparison to the language. Early Human Develop. 2003; 75:55-69.

14. Baeck HE, Souza MN. Longitudinal study of the fundamental frequency of hunger cries along the first 6 months of healthy babies. J Voice. 2007; 21(5):551- 9 .

15. Behlau MS. Uma análise das vogais do português brasileiro falado em São Paulo: perceptual, espectrográfica de formantes e computadorizada de frequência fundamental. [dissertação] São Paulo (SP): Escola Paulista de Medicina; 1984.

16. Huber JE, Stathopoulos ET, Curione GM, Ash TA, Johnson K. Formants of children, women and men: the effects of vocal intensity variation. J Acoust Soc Am. 1999; 106(3):1532-42.

17. Cappellari VM, Cielo CA. Características vocais acústicas de crianças pré-escolares. Rev Bras Otorrinolaringol. 2008; 74(2):265-72.

18. Nicollas R, Garrel R, Ouaknine M, Giovanni A, Nazarian B, Triglia, JM. Normal voice in children between 6 and 12 years of age: database and nonliner analysis. J Voice. 2008; 22(6):671-5. 
19. Baker S, Weinrich B, Bevington M, Schroth K, Schroeder E. The effect of task type on fundamental frequency in children. Int J Pediatr Otorhinolaryngol. 2008; 72:885-9.

20. Braga JN, Oliveira DSF, Sampaio TMM. Frequência fundamental da voz de crianças. Rev. CEFAC. 2009; 11(1):119-26.

21. Schott TCA, Sampaio MM, Oliveira DSF. Frequência fundamental de crianças da cidade de Niterói. Rev. CEFAC. 2009; 12(2):290-5.

22. Pinho SMR, Pontes P. Desvendando os segredos da voz. Músculos intrínsecos da laringe e dinâmica vocal. vol. 1. Rio de Janeiro: Revinter; 2008.

23. Pinho SMR. Avaliação e tratamento da voz. In: Pinho SMR. Fundamentos em fonoaudiologia. Tratando os distúrbios da voz. 2. ed. Rio de Janeiro: Guanabara Koogan; 2003. p. 3-40.
24. Vanzella TH. Normatização dos parâmetros acústicos vocais em crianças em idade escolar. [dissertação] Ribeirão Preto (SP): Universidade de São Paulo; 2006.

25. Busby PA, Plant GL. Formant frequency values of vowels produced by preadolescent boys and girls. J Acoust Soc Am. 1995; 97(4):2603-6.

26. Glaze LE, Bless DM, Milenkovic P, Susser RD. Acoustic characteristics of children's voice. J Voice. 1988; 2(4):312-9.

27. Lee S, Potamianos A, Narayanan S. Acoustic of children's speech: developmental changes of temporal and spectral parameters. J Acoustic Soc Am. 1999; 105(3):1455-68.

28. Perry TL, Ohde RN, Ashmead DH. The acoustic bases for gender identification from children's voices. J Acoustic Soc Am. 2001; 109(6):2988-98.

RECEBIDO EM: 21/09/2009

ACEITO EM: 23/12/2009

Endereço para correspondência:

Flávia Viegas

Rua Chaves Pinheiro, 58 sl. 101

Rio de Janeiro - RJ

CEP: 20771-470

E-mail: fdviegas@gmail.com 